\title{
SIMULATING THE DYNAMICS OF ENTANGLED POLYMERS USING THE KINETIC THEORY DESCRIPTION OF THE DOI-EDWARDS MODEL
}

\author{
B. Mokdad \\ Laboratoire de Rhéologie \\ INPG / UJF I CNRS (UMR 5520) \\ 1301 rue de la piscine, BP 53 Domaine universitaire \\ 38041 Grenoble Cedex 9, France \\ Bechir.Mokdad@ujf-grenoble.fr
}

\author{
A. Ammar \\ Laboratoire de Rhéologie \\ INPG / UJF / CNRS (UMR 5520) \\ 1301 rue de la piscine, BP 53 Domaine universitaire \\ 38041 Grenoble Cedex 9, France \\ Amine.Ammar@ujf-grenoble.fr
}

\author{
F. Chinesta \\ LMSP (Laboratoire de Mécanique des Systèmes et des Procédés) \\ UMR CNRS - ENSAM - ESEM \\ 151 Boulevard de l'Hôpital, F-75013 Paris, France \\ francisco.chinesta@paris.ensam.fr
}

\begin{abstract}
In this work, the model of Doi-Edwards with independant alignment approximation describing the dynamics of polymer melts is simulated. The main aim of this work is the analysis of some new simulation techniques operating on the FokkerPlanck equation related to that model. For this purpose we consider the kinetic theory description of the Doi-Edwards model, implemented in the 2D and 3D cases under shear and elongational flows. The Fokker Planck equation which governs the evolution of the distribution function involves two variables: the tube orientation (described by a unit vector defining the unit surface in 3D and the unit circle in 2D) and the coordinate that locates the segment tube on the molecular chain, taking values in the unit interval. To separate both variables during the problem resolution we make use of the Alternating Direction Implicit method (ADI) which allows reducing the computation time and efforts.
\end{abstract}

A model reduction technique is also proposed and analyzed. It consists of considering an optimal representation basis which is constructed during the problem resolution. Thus, a reduced number of approximation functions, now defined in the whole domain, are enough to describe the solution evolution during the entire time interval considered in the simulation, with significant CPU time savings.

Keywords: Polymer melt, Kinetic theory, DoiEdwards model, Reptation, Alternating Direction Implicit Mehod, Model reduction, Karhunen-Loeve decomposition, Krylov subspaces

\section{INTRODUCTION: DOI-EDWARDS MODEL WITH INDEPENDENT ALIGNMENT APPROXIMATION}

In most polymer processing operations such as injection molding, film blowing and extrusion, the polymers are in the molten state. A widely applied class of molecular-based models for concentrated polymer solutions and melts relies on the notion of reptational motion: Doi-Edwards model (M. Doi and S. F. Edwards., 1978) being one a such model.

The key idea of this model is application of the reptation mechanism introduced by De-Gennes (P. G. De Gennes., 1971) to a tube (along which the molecule can move) in order to describe the viscoelastic behaviour of entangled polymers. The molecule is described as sliding or reptating through a tube whose contours are defined by the locus of entanglements with neighbouring molecules. The motion of a molecular chain in any other direction than the one defined by the tube axis is strongly 
restricted, except at both tube ends, where it can move in any possible direction. The tube moves itself due to two mechanisms: (i) by means of the motion of the central chain itself, which partially leaves its original tube, for extending it in other directions, and (ii) by the fluctuation induced by the motions of the neighbour chains defining the tube lateral border. In addition to the reptation mechanism, the Doi-Edwards model assumes affine tube deformation induced by the macroscopic flow as well as a complete chain, but neglects other phenomena like the stretch of the chain and the Convective Constraint Release. Within this reptation picture and these assumptions, the dynamics of a single segment is given by:

$\frac{d \underline{u}}{d t}=(\underline{\underline{I}}-\underline{u} \otimes \underline{u}) \operatorname{grad} \underline{v} \underline{u}$

Where $\underline{u}$ is the unit vector describing the orientation of the tube segment, grad $\underline{v}$ the velocity gradient and $\underline{\underline{I}}$ the unit tensor. The distribution function $\psi$ is such that $\psi(\underline{u}, s, x, t) d \underline{u} d s$ represents the joint probability that at time $\mathrm{t}$ and position $\mathrm{x}$ a tube segment has an orientation in the interval $[\underline{u}, \underline{u}+d \underline{u}]$ and contains the chain segment labelled in the interval $[s, s+d s]$. Thus, the configuration space is $\Omega=\mathrm{B}(0,1) \times[0,1]$, where $\mathrm{B}(0,1)$ is the surface of the unit sphere centered at the origin. The Fokker-Planck equation related to the Doi-Edwards model is the convection-diffusion equation that governs the conservation balance of the distribution function:

$\frac{d \psi}{d t}=-\frac{\partial}{\partial \underline{u}}[[(\underline{I}-\underline{u} \otimes \underline{u}) \operatorname{grad} \underline{v} \underline{u}] \psi]+\frac{1}{\pi^{2} \tau_{d}} \frac{\partial^{2} \psi}{\partial s^{2}}$

Where $\frac{d}{d t}$ represents the material derivative, $\tau_{d}$ is the disengagement time, namely the characteristic time for a chain to come out the tube by reptation. We can define the diffusion coefficient related to the s-coordinate as $D_{r}=\frac{1}{\pi^{2} \tau_{d}}$. To solve the FokkerPlanck equation, one needs to prescribe appropriate boundary conditions at the tube borders $s=0$ and $s=1$ (the orientation coordinate being defined on the unit sphere does not require any boundary condition). In general, as previously indicated, an isotropic orientation distribution is prescribed at both ends, which reads:

$$
\psi(\underline{u}, s=0, x, t)=\psi(\underline{u}, s=1, x, t)=\frac{1}{4 \pi} \delta(\|\underline{u}\|-1)
$$

where $\delta$ is the Dirac delta distribution. Knowing the distribution function, the stress can be computed from:

$$
\underline{\underline{\tau}}_{p}=G \int_{0}^{1} \int_{B(0,1)} \underline{u} \otimes \underline{u} \psi d \underline{u} d s
$$

where $\mathrm{G}$ is an elastic modulus and $\underset{=}{\tau}$ is the polymer stress tensor.

\section{RESOLUTION STRATEGY: ALTERNATING DIRECTION IMPLICIT}

The alternating direction implicit method, ADI, (Jr. J. Douglas and J. E. Gunn.,1964) has been found to be effective for many problems, and generally has a faster convergence rate than SOR strategies. The idea is an alternating resolution of the problems defined in each coordinate. Thus, the ADI strategy instead of solving the 2D (respectively 3D) problem, solves a succession of two (respectively three) one-dimensional problems. The major advantages of the ADI method are: (i) it is unconditionally stable for some operator due to its implicit character; (ii) it can be applied for solving numerous multidimensional problems (in moderate dimensions).

The first step to define a finite-difference scheme for solving a partial differential equation is to discretize the continuous space domain with a grid, whose number of nodes depends on the solution to be approximated. In what follows we are considering only homogeneous flows, which allows to write $\psi(\underline{u}, s, t)$ (that does not depend on the physical coordinates). The degree of freedom related to a grid point $(\mathrm{i}, \mathrm{j}, \mathrm{n})$ is $\psi\left(\underline{u}_{i}, s_{j}, t_{n}\right)$ that is usually denoted by $\psi_{i, j}^{n}$.

Now, at each time step $n$, the alternating directions method solves (in the 2D case) two steps:

Step I: U-updating:

$$
\begin{aligned}
\frac{\psi_{i, j}^{n+\frac{1}{2}}-\psi_{i, j}^{n}}{\Delta t / 2}=-E_{1}\left(u_{i}\right)\left[\beta\left(\frac{\psi_{i, j}^{n+\frac{1}{2}}-\psi_{i-1, j}^{n+\frac{1}{2}}}{\Delta u}\right)+(1-\beta)\left(\frac{\psi_{i+1, j}^{n+\frac{1}{2}}-\psi_{i, j}^{n+\frac{1}{2}}}{\Delta u}\right)\right] \\
-E_{0}\left(u_{i}\right) \psi_{i, j}^{n+\frac{1}{2}}+D_{r}\left[\frac{\psi_{i, j-1}^{n}-2 \psi_{i, j}^{n}+\psi_{i, j+1}^{n}}{(\Delta s)^{2}}\right]
\end{aligned}
$$

Step II: S-updating: 


$$
\begin{aligned}
\frac{\psi_{i, j}^{n+1}-\psi_{i, j}^{n+\frac{1}{2}}}{\Delta t / 2}=-E_{1}\left(u_{i}\right) & {\left[\beta\left(\frac{\psi_{i, j}^{n+\frac{1}{2}}-\psi_{i-1, j}^{n+\frac{1}{2}}}{\Delta u}\right)+(1-\beta)\left(\frac{\psi_{i+1, j}^{n+\frac{1}{2}}-\psi_{i, j}^{n+\frac{1}{2}}}{\Delta u}\right)\right] } \\
& -E_{0}\left(u_{i}\right) \psi_{i, j}^{n+\frac{1}{2}}+D_{r}\left[\frac{\psi_{i, j-1}^{n+1}-2 \psi_{i, j}^{n+1}+\psi_{i, j+1}^{n+1}}{(\Delta s)^{2}}\right]
\end{aligned}
$$

Due to the convection-diffusion character of the equation to be solved in the first step an appropriate stabilization is needed to avoid numerical instabilities induced by the convection term. In our simulations we considered a streamline upwinding, where the upwinding parameter $\beta$ approaches to 1 when advection becomes dominant and $E_{1}\left(u_{i}\right)>0$ (respectively to zero for dominant advection and $\left.E_{1}\left(u_{i}\right)<0\right)$. When the diffusion term is dominant $\beta$ approaches to 0.5 and then, both terms affected by the beta coefficients are equivalent to the centred finite difference.

In the 2D case, step I, requires the resolution of a system of equations. This resolution must be done as many times as the number of nodes of s-axis discretization (we exclude the nodes located at the border where the boundary condition is prescribed). The linear system can be expressed by:

\section{$\underline{\underline{M}} \underline{\psi}+\underline{\underline{G}} \underline{\psi}=\underline{S}$}

The difference between the $2 \mathrm{D}$ and the $3 \mathrm{D}$ cases is that in the first case, vector $\underline{u}$ can be easily described by the scalar $\varphi(0 \leq \varphi \leq 2 \pi)$ whereas in the 3D case $\underline{u}$ needs two scalars, $\varphi$ and $\theta$ if one considers polar coordinates $(0 \leq \varphi \leq 2 \pi$ and $0 \leq \theta \leq \pi)$.

\section{NUMERICAL RESULTS}

The distribution function is represented in the space of configurations and shows a periodic evolution according to the space orientation $(\underline{u}(\varphi)$ in the $2 \mathrm{D}$ case and $\underline{u}(\varphi, \theta)$ in the $3 \mathrm{D}$ case) and a parabolic evolution according to the curvilinear coordinate (s), see Figures 1, 2, 3 and 4, proving, as expected, that segments located in the central region are more stretched, and their orientations more constrained, than the ones located in the borders neighbourhood.

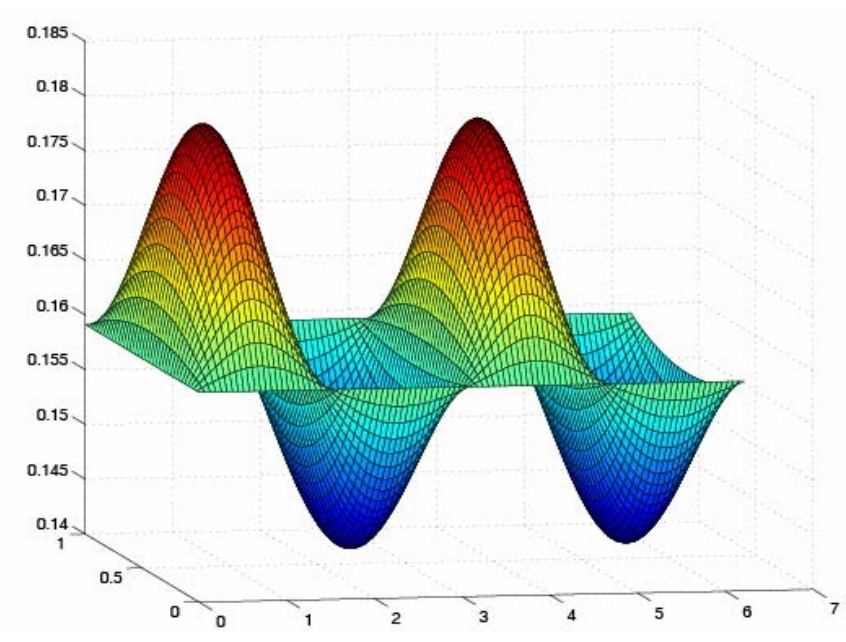

Figure 1 Evolution of the distribution function in a 2D shear flow case with $\mathrm{Dr}=1$ and $\mathrm{We}=1$.

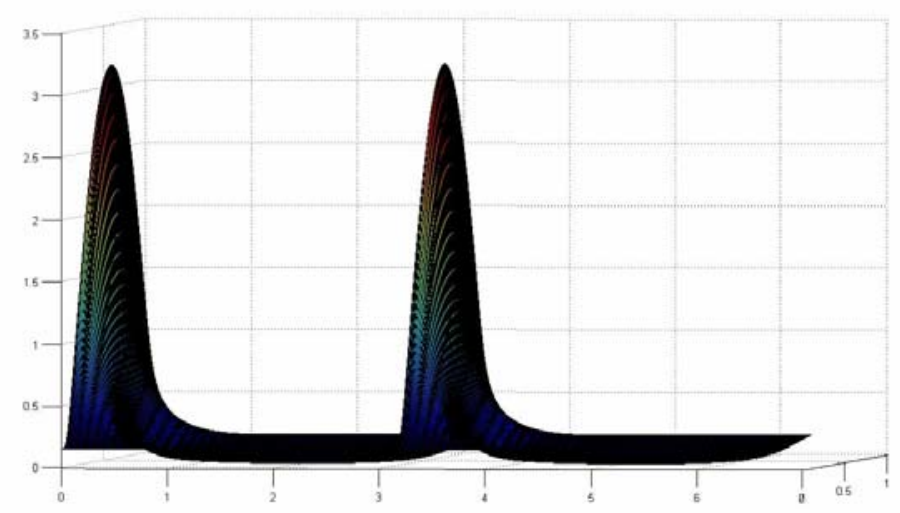

Figure 2 Evolution of the distribution function in a 2D shear flow case with $\mathrm{Dr}=1$ and $\mathrm{We}=100$.

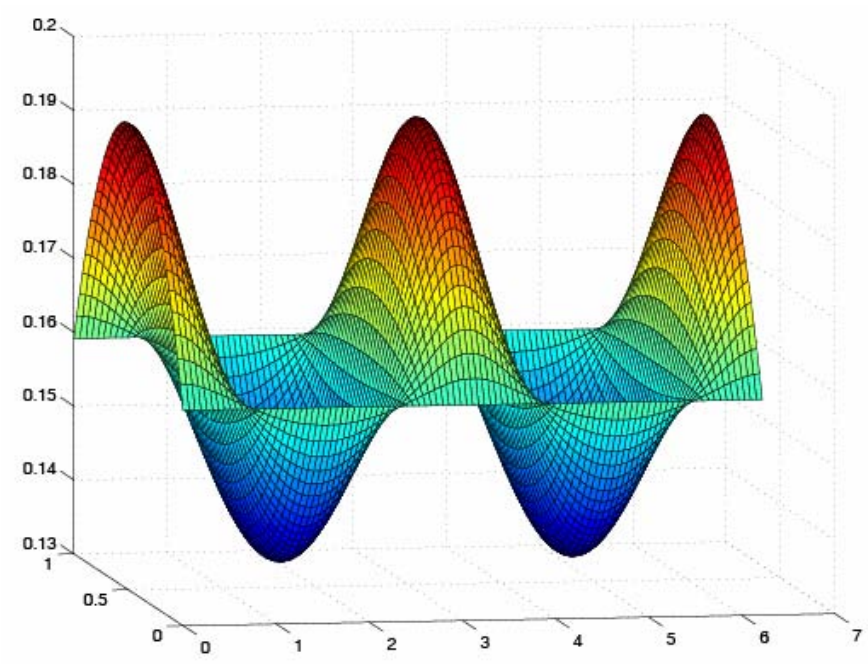

Figure 3 Evolution of the distribution function in a $2 \mathrm{D}$ elongation flow case with $\mathrm{Dr}=1$ and $\mathrm{We}=1$.

\section{D simulations:}




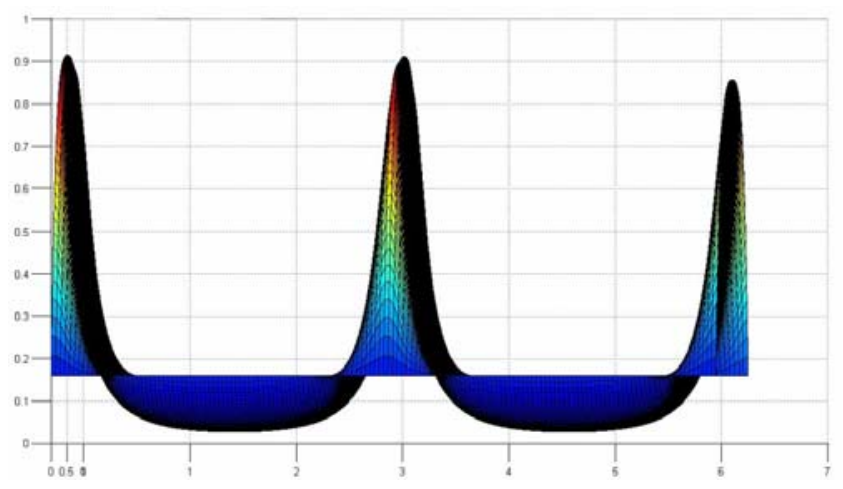

Figure 4 Evolution of the distribution function in a 2D elongation flow case with $\mathrm{Dr}=1$ and $\mathrm{We}=50$.

We can also notice at these figures that as the Weissenberg number increases the peaks become more prominent and more localized noticing that the chain is the more and more sollicitated.

\section{D simulations:}

In this case, due to the symmetry of the chain, we consider only a half, Figure 5.

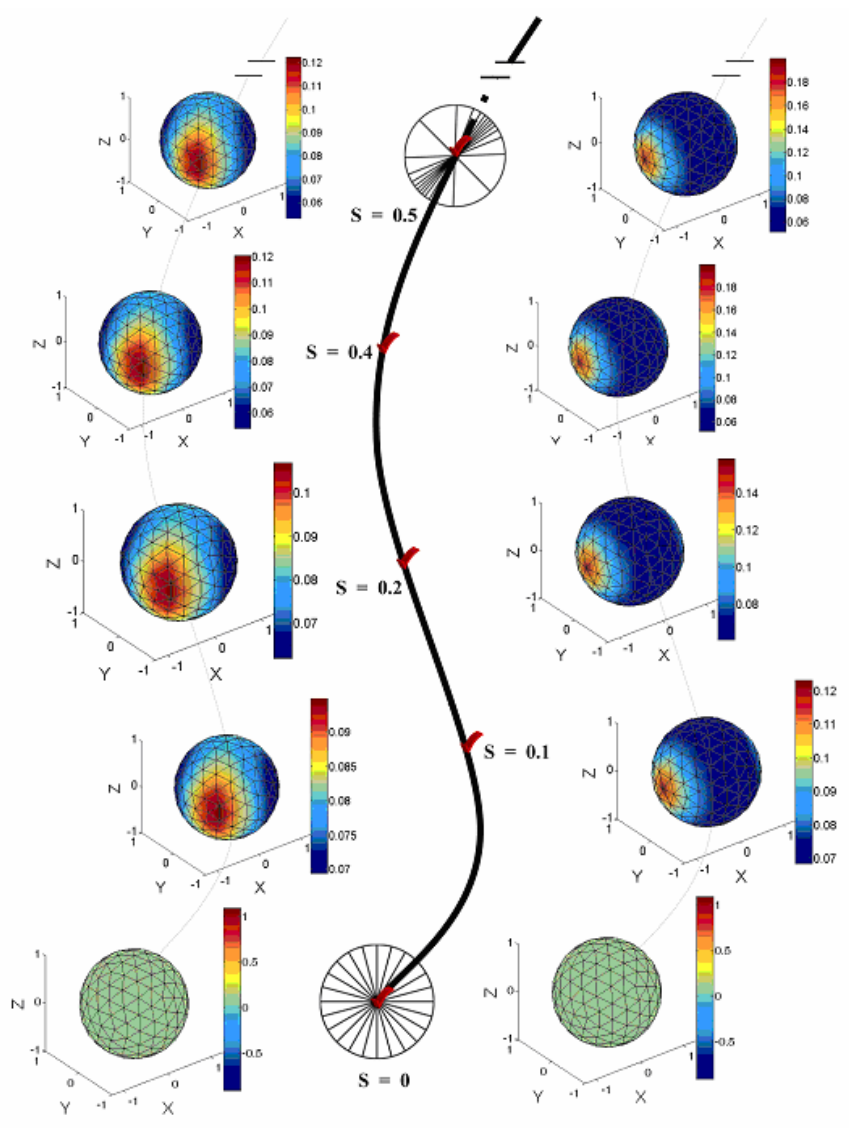

Figure 5 Evolution of the distribution function in a $3 \mathrm{D}$ case with $\mathrm{Dr}=1$ and $\mathrm{We}=1$ : (left) Shear flow

(right) Elongation flow.
The micro-macro modeling is done by the calculation of the stress tensor, and then the shear and elongation viscosities. The steady shear viscosity, depicted in Figure 6, decreases as the shear rate increases (shear thinning behaviour). At high shear rates, the asymptotic viscosity curve related to the Doi-Edwards model possesses a slope of $-3 / 2$ (in the double logarithmic presentation), higher than the -1 slope (also indicated in that plot) noticing for the instability of that model.

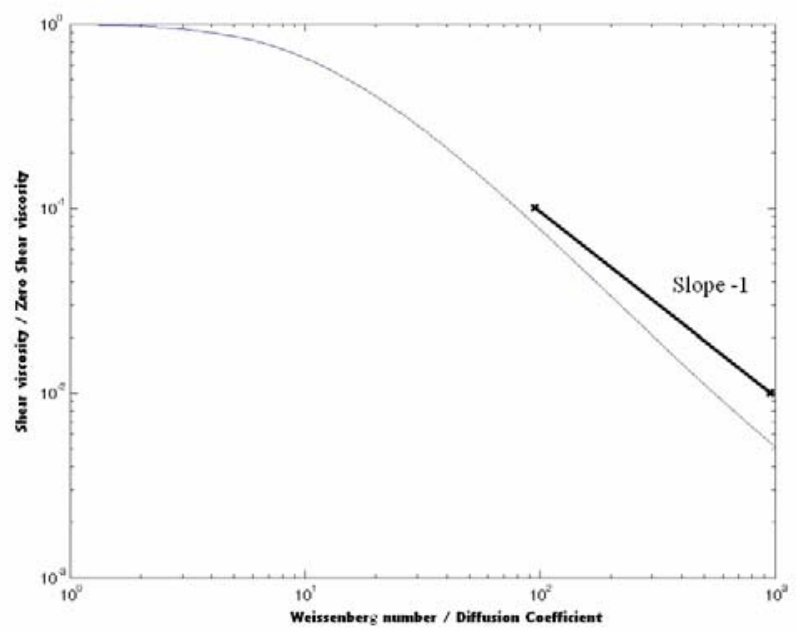

Figure 6 Steady shear viscosity, 2D case.

Besides, we observe that the shear stress shows transient overshoots in the start-up shear flow at low shear rates, which is one of the major features that the Doi-Edwards model is not able to predict.

\section{MODEL REDUCTION}

The goal of the reduction model is to define an optimal approximation subspace able to represent accurately the whole field evolution, in our case the time evolution of the distribution function. As the number of approximation function is much lower than the number of grid points, the associated linear system is of low dimension and significant CPU time savings can be attained. The new shape functions are defined in the whole domain in an appropriate manner (the most characteristic functions related to the model solution) (A.Ammar et al., 2005). The construction of those new approximation functions is done with 'a priori' approach.

In this section we are going to apply the ideas just introduced to the discrete problem resulting from the discretisation of the FokkerPlanck equation that we can express in the matrix form: 
$\underline{\underline{M}} \dot{\psi}+\underline{\underline{G}} \underline{\psi}=\underline{S}$

whose implicit time discretization results:

$\underline{\psi}_{t+\Delta t}=(\underline{\underline{M}}+\Delta t \underline{\underline{G}})^{-1} \underline{\underline{M}} \underline{\psi}_{t}+\Delta t(\underline{\underline{M}}+\Delta t \underline{\underline{G}})^{-1} \underline{S}$

We consider that the probability distribution have been accurately described in $\left[0, t_{\alpha}\right]$. We assume that at time $t_{\alpha}$ the reduced approximation basis is given by $\underline{\underline{B}}^{(n)}$. Moreover, at certain times $t_{p}$, with $t_{p} \leq t_{\alpha}$, the solution is assumed known and defined by the reduced vectors $\underline{\xi}_{p}^{(n)}$. Knowing $\underline{\xi}_{p}^{(n)}$, the finite element description of $\psi$ at time $t_{p}$ results: $\underline{\psi}^{p}=\underline{\underline{B}}^{(n)} \underline{\xi}_{p}^{(n)}$. We can assume that the first approximation basis $\underline{\underline{B}}^{(0)}$ contains a single vector that corresponds with the initial probability $\psi^{0}$. Now, we compute the evolution of $\psi$ in $\left[t_{\alpha}, t_{\beta}\right]$ solving the reduced form related to implicit scheme:

$$
\begin{aligned}
& \left(\underline{\underline{B}}^{(n)}\right)^{T} \underline{\underline{B}}^{(n)} \underline{\underline{\xi}}_{t+\Delta t}^{(n)}= \\
& =\left(\underline{\underline{B}}^{(n)}\right)^{T}(\underline{\underline{M}}+\Delta t \underline{\underline{G}})^{-1} \underline{\underline{M}} \underline{\underline{B}}^{(n)} \underline{\underline{\xi}}_{t}^{(n)}+\Delta t\left(\underline{\underline{B}}^{(n)}\right)^{T}(\underline{\underline{M}}+\Delta t \underline{\underline{G}})^{-1} \underline{\underline{S}}
\end{aligned}
$$

The new reduced approximation basis $\underline{\underline{B}}^{(n+1)}$ is defined by adding to the significant information extracted from $\underline{\xi}_{p}^{(n)}, \forall p \leq \alpha$, some Krylov's subspaces computed at $t_{\beta}$. We are going to explicit the construction of $\underline{\underline{B}}^{(n+1)}$.

We define the matrix $\underline{\underline{Q}}$ which contains the reduced vectors $\underline{\xi}_{p}^{(n)}, \forall p \leq \alpha$. Now, we solve the eigenproblem related to the Karhunen-Loeve decomposition, given by:

$\underline{\underline{Q}} \underline{\underline{Q}}^{T} \underline{\vartheta}=\lambda \underline{\vartheta}$

Whose solution results in $\alpha$ couples $\left(\underline{\vartheta}_{k}, \lambda_{k}\right)$, where we assume that eigenvalues are ordered $\lambda_{1} \geq \lambda_{2} \ldots . \geq \lambda_{k}$, and we select the eigenvectors $\underline{\vartheta}_{k}$ related to the eigenvalues verifying $\lambda_{k} \geq 10^{-8} \lambda_{1}$, that constitute the columns of matrix $\underline{\underline{V}}$. Now we can write:
$\underline{\underline{\tilde{B}}}^{(n+1)}=\left(\underline{\underline{V}}^{\underline{B^{(n+1)}}}\right)$

Obviously, the change in the reduced approximation basis implies a change in the expression of the reduced vectors $\underline{\xi}_{p}^{(n)}, \forall p \leq \alpha$. For this purpose one could enforce:

$$
\underline{\underline{\tilde{B}}}^{(n+1)} \underline{\tilde{\xi}}_{p}^{(n+1)}=\underline{\underline{B}}^{(n)} \underline{\xi}_{p}^{(n)}
$$

which it results:

$$
\begin{aligned}
& \left(\underline{\underline{\tilde{B}}}^{(n+1)}\right)^{T} \underline{\underline{\tilde{B}}}^{(n+1)} \underline{\xi}_{p}^{(n+1)}=\left(\underline{\underline{\tilde{B}}}^{(n+1)}\right)^{T} \underline{\underline{B}}^{(n)} \underline{\xi}_{p}^{(n)} \Rightarrow \\
& \underline{\tilde{\xi}}_{p}^{(n+1)}=\left(\left(\underline{\underline{\tilde{B}}}^{(n+1)}\right)^{T} \underline{\underline{\tilde{B}}}^{(n+1)}\right)^{-1}\left(\underline{\underline{\tilde{B}}}^{(n+1)}\right)^{T} \underline{\underline{B}}^{(n)} \underline{\xi}_{p}^{(n)} \\
& \forall p \leq \alpha
\end{aligned}
$$

Now, the updated basis consist of $\underline{\underline{\tilde{B}}}^{(n+1)}$ completed with some Krylov subspaces generated from the residual $\underline{R}$ at time $t_{\beta}$ :

$$
\underline{\underline{B}}^{(n+1)}=\left(\underline{\underline{V}}^{(n)}, \underline{R}, \cdots\right)
$$

where the residual is computed according to:

$$
\begin{aligned}
& \underline{R}=\underline{\underline{B}}^{(n)} \underline{\xi}_{t_{\beta}}^{(n)}- \\
& -(\underline{\underline{M}}+\Delta t \underline{\underline{G}})^{-1} \underline{\underline{M}}_{\underline{\underline{B}}}^{(n)} \underline{\underline{\xi}}_{t}^{(n)}+\Delta t\left(\underline{\underline{B}}^{(n)}\right)^{T}(\underline{\underline{M}}+\Delta t \underline{\underline{G}})^{-1} \underline{\underline{S}}
\end{aligned}
$$

In order to analyze the capabilities of this technique we consider the 3D Doi-Edwards model. The technique just described allows to define a reduced approximation basis that is able to represent the entire time evolution of the distribution using only 8 functions, and then only 8 degrees of freedom (at each time step one needs to perform the inversion of a matrix of size $8 \times 8$ ) instead the 642 nodes considered in the coarse mesh illustrated in Figure 7. The computed results are in agreement with the ones obtained using the ADI strategy. 

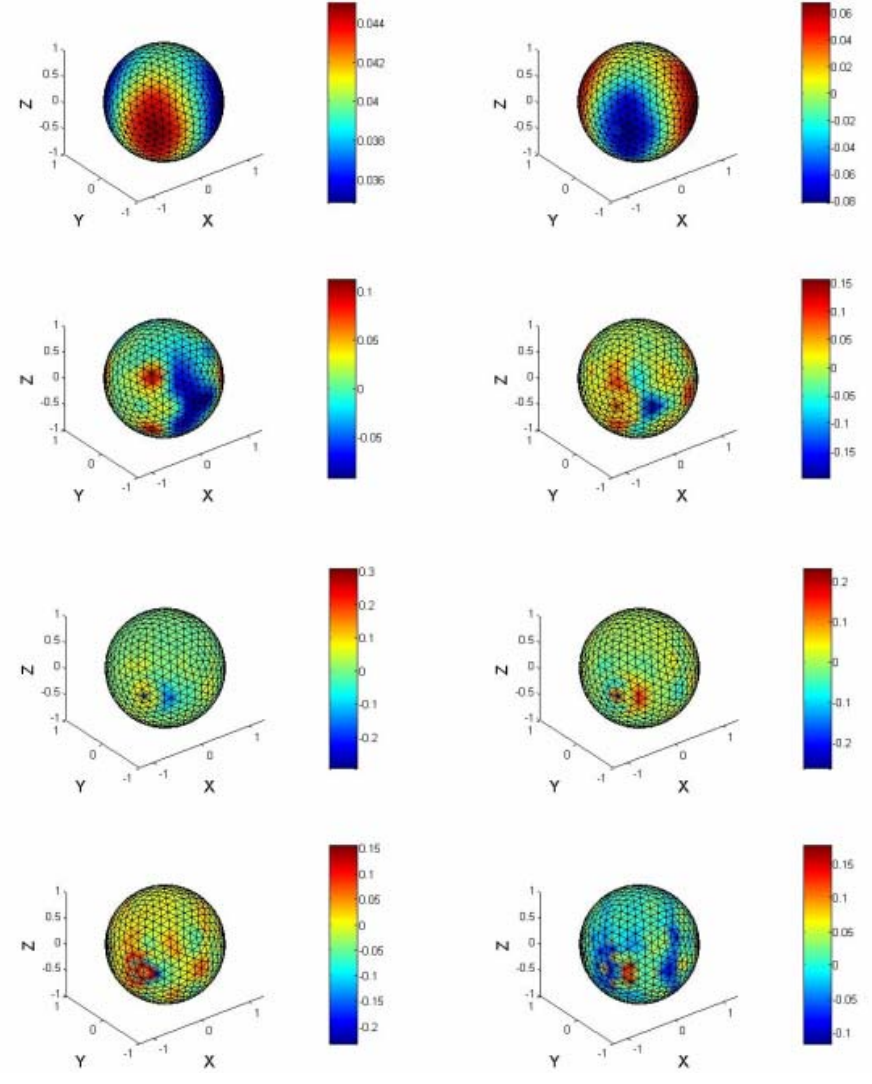

Figure 7 The eight most significatives reduced basis functions:3D case.(up-left) First most significative reduced basis function; (up-right) Second most significative reduced basis function ;...; (down-left) Seventh most significative reduced basis function;(down-right) Eighth most significative reduced basis function.

\section{CONCLUSIONS}

In this paper, the Doi-Edwards model with independant alignment approximation has been analyzed, by solving the associated Fokker-Planck equation using two different numerical strategies that combine great accuracy and significant CPU time savings. The first strategy makes use a decoupling between the different model coordinates during the discretization, and the second one is based on the construction of a reduced approximation basis able to represent accurately the entire time evolution of the distribution function.

At present we are developing a second generation of model reduction techniques based on the use of separated representations of fields combined with tensor product approximation spaces. These techniques have been applied in some of our former works (A.Ammar et al., 2006) for solving dumbbells modes (FENE, MBS, ...) and seem to be excellent candidates for treating models describing entangled polymers based on the reptation motion or in the molecular networks.

In any case, more realistic models are being considered to avoid the previously referred overshoot in the shear viscosity, being an appealing model the one propose by Ottinger (H. C. Ottinger., 2000).

\section{REFERENCES}

A. Ammar, B. Mokdad, F. Chinesta and R. Keunings., "A Fast Solver for some classes of Multidimensional Partial Differential Equations Encountered in Kinetic Theory Modeling of Complex Fluids", submitted to J. Non-Newtonien Fluid Mech. (2006).

A. Ammar and F. Chinesta., "A Particle strategy for solving the Fokker-Planck equation modelling the fiber orientation distribution in steady recirculating flows involving short fiber suspensions", Lectures Notes on Computational Science and Engineering, Springer, 43, 2005, 1-16.

A. Ammar, D. Ryckelynck, F. Chinesta and R. Keunings., "On the Reduction of Kinetic Theory Models Related to Finitely Extensible Dumbbells", submitted to J. Non-Newtonien Fluid Mech. (2005).

F. Chinesta, G. Chaidron and A. Poitou., "On the solution of the Fokker-Planck equation in steady recirculating flows involving short fiber suspensions”, J. Non-Newtonien Fluid Mech, 113 (2003) 97-125.

C. F. Curtiss and R. B. Bird., "A kinetic theory for polymer melts. I. The equation for the single-link orientational distribution function", J. Chem. Phys., 74 (1981-a) 2016-2025.

C. F. Curtiss and R. B. Bird., "A kinetic theory for polymer melts. II. The stress tensor and the rheological equation of state”, J. Chem. Phys., 74 (1981b) 2026-2033.

J. des Cloizeaux., "Double reptation vs simple reptation in polymer melts“, Europhys. Lett. 5 (1988) 437-442.

P. G. De Gennes, "Reptation of a polymer chain in the presence of fixed obstacles", J. Chem. Phys. 55, 572-579 (1971).

M. Doi and S. F. Edwards, "The theory of polymer dynamics”, Oxford Science Publications.

M. Doi and S. F. Edwards, "Dynamics of concentrated polymer systems, Part I”, J. C. S Faraday II, 74 (1978) 1789-1801.

M. Doi and S. F. Edwards, "Dynamics of concentrated polymer systems, Part II”, J. C. S Faraday II, 74 (1978) 1802-1817.

M. Doi and S. F. Edwards, "Dynamics of concentrated polymer systems, Part III”, J. C. S Faraday II, 74 (1978) 1818. 
M. Doi, "Explanation for the 3.4-power law for viscosity of polymeric liquids on the basis of the tube model”, J. Polym. Sci. Polym. Phys. Ed, 21 (1983) 667684.

Jr. J. Douglas and J. E. Gunn., “A general formulation of alternating direction methods - Part I: Parabolic and hyperbolic problems", Numer. Math, 6 (1964) 428-453.

J. Fang, M. Kroger and H. C. Ottinger., "A thermodynamically admissible reptation model for fast flows of entangled polymers. II. Model predictions for shear and extensional flows”, J. Rheol., 40 (2000) 12931318.

R. S. Graham, A. E. Likhtman, T. C. B. Mcleish and S. T. Milner "Microscopic theory of linear entangled polymer chains under rapid deformation including chain stretch and convective constraint release”, J. Rheol., 47 (2003) 1171-1200.

M. A. Hulsen , "Mico-macro simulation of polymer solutions and melts", Delft University of Technology.

G. B. Jeffery., "The motion of ellipsoidal particles immersed in a viscous fluid”, Proc. Roy. Soc. London, Ser A 102 (1922) 161-179.

B. Jourdain, T. Lelièvre, and C. Le Bris., "Numerical analysis of micro-macro simulations of polymeric fluid flows : a simple case”, Math. Models and Methods in Applied Sciences, 12 (2002) 1205-1243.

R. Ketzmerick and H. C. Ottinger, "Simulation of a non-Markovian process modelling contour length fluctuation in the Doi-Edwards model”, Continuum Mech. Thermodyn. 1, (1989) 113-124.

R. Keunings , "Finite elements methods for integral viscoelastic fluids", Binding D M and Walters (Eds.), British Society of Rheology, Aberystwyth, Wales (2003) 167-195.

R. Keunings., "Fundamentals of computer modeling for polymer processing”, Chapter simulation of viscoelastic fluid flow, (1989) 402-470.

R. Keunings., "Micro-macro methods for the multiscale simulation of viscoelastic flow using molecular models of Kinetic theory”, Rheology Reviews 2004, D. M. Binding and K. Walters (Eds.), British Society of Rheology. fluid flow, (1989) 402-470.

M. Kroger., "Simple models for complex nonequilibrum fluids”, Physics Reports, 390 (2004) 453551.

R. G. Larson., "Constitutive equations for polymer melts and solutions", Butterworth, Boston.

A. Leygue, A. N. Beris, R. Keunings., "A constitutive equation for entangled linear polymers inspired by reptation theory and consistent with nonequilibrium thermodynamics”, J. Non-Newtonien Fluid Mech, 101 (2001) 95-111.

E. Rincon, A. E. Chavez and O. Manero., "Rheological modelling of complex fluids : A transient network model with microstates”, J. Non-Newtonian fluid Mech, 131(2005)64-77.
G. Marrucci., "The Doi-Edwards model without independant alignment “, J. Non-Newtonien Fluid Mech, 21 (1986) 329-336.

D.W. Mead, R. G. Larson and M. S. Doi., "A molecular theory for fast flows of entangled polymers", Macromolecules., 31 (1998) 7895-7914.

N. P. T. O'connor and R. C. Ball., "Confirmation of the Doi-Edwards Model”, Macromolecules., 25 (1992) 5677-5682.

H. C. Ottinger., "Thermodynamically admissible reptation models with anisotropic tube cross sections and convective constraint release", J. Non-Newtonian Fluid Mech., 89 (2000) 165-185.

H. C. Ottinger., "A thermodynamically admissible reptation model for fast flows of entangled polymers”, J. Rheol., 43 (1999) 1461-1493.

H. C. Ottinger., "Stochastic processes in polymeric fluids : tools and examples for developing simulation algorithms”, Springer, Berlin (1996).

D. W. Peaceman and Jr. H. H. Rachford., "The numerical solution of parabolic and elliptic differential equations”,J. Soc. Ind. Appl. Math, 3(1955)28-41.

E. A. J. F. Peters, A. P. G Van Heel, M. A. Hulsen and B. H. A. A Van den Brule, "Generalization of the deformation field method to simulate advanced reptation models in complex flows”, J. Rheol., 44 (2000) 811-829.

D. Reyckelynck, F. Chinesta, E. Cueto and A. Ammar, "On the priori model reduction : overview and recent developpements", Archives of Computational Methods in Engineering, In press.

C. Tsenoglou, "Viscoelasticity of binary homopolymer blends”, ACS Polym. Preprints 28, (1987) 185-186.

A. P. G Van Heel, M. A. Hulsen and B. H. A. A Van den Brule, "Simulation of the Doi-Edwards model in complex flows”, J. Rheol., 43(1999)1239-1260.

P. Wapperom and R. Keunings, "Numerical simulation of branched polymer melts in transient complex flow using pom-pom models”, J. NonNewtonien Fluid Mech, 97 (2001) 267-281.

P. Wapperom and R. Keunings, "Simulation of linear melts in transient complex flow", J. NonNewtonien Fluid Mech, 95 (2000) 67-83. 